\title{
Size effects in ultrathin epitaxial ferroelectric heterostructures
}

\author{
V. Nagarajan, ${ }^{\text {a) }}$ S. Prasertchoung, T. Zhao, H. Zheng, J. Ouyang, and R. Ramesh ${ }^{\text {b) }}$ \\ Materials Research Science and Engineering Center, University of Maryland, College Park, \\ Maryland 20742 \\ W. Tian and X. Q. Pan \\ Department of Materials Science and Engineering, University of Michigan, Ann Arbor, Michigan 48109 \\ D. M. Kim and C. B. Eom \\ Department of Materials Science and Engineering, University of Wisconsin-Madison, Madison, \\ Wisconsin 53706 \\ H. Kohlstedt and R. Waser \\ Institut für Festkörperforschung and CNI, Forschungzentrum Jülich, D-52425 Jülich, Germany
}

(Received 28 January 2004; accepted 1 May 2004; published online 10 June 2004)

\begin{abstract}
In this letter we report on the effect of thickness scaling in model $\mathrm{PbZr}_{0.2} \mathrm{Ti}_{0.8} \mathrm{O}_{3}(\mathrm{PZT}) / \mathrm{SrRuO}_{3}$ heterostructures. Although theoretical models for thickness scaling have been widely reported, direct quantitative experimental data for ultrathin perovskite $(<10 \mathrm{~nm})$ films in the presence of real electrodes have still not been reported. In this letter we show a systematic quantitative experimental study of the thickness dependence of switched polarization in (001) epitaxial PZT films, 4 to $80 \mathrm{~nm}$ thick. A preliminary model based on a modified Landau Ginzburg approach suggests that the nature of the electrostatics at the ferroelectric-electrode interface plays a significant role in the scaling of ferroelectric thin films. (0) 2004 American Institute of Physics.
\end{abstract}

[DOI: $10.1063 / 1.1765742]$

The effect of thickness scaling in ferroelectrics has recently been of immense interest. ${ }^{1-12}$ As the dimensions (both lateral and vertical direction) of the perovskite layer decreases, the fundamental question of size dependence becomes crucial. From a theoretical point of view, two models have been traditionally used to describe size effects, namely an "intrinsic" effect"10,13 and a "depoling field" effect. ${ }^{4,5,14,15}$ Experimentally Tybell et al. ${ }^{8}$ qualitatively showed that even a 4-nm-thick epitaxial $\mathrm{PbZr}_{0.2} \mathrm{Ti}_{0.8} \mathrm{O}_{3}$ (PZT) film on $\mathrm{Nb}: \mathrm{STO}$ is ferroelectric. However a direct experimental quantification of the ferroelectric polarization, particularly for films in the sub-10 nm thickness range has not been reported. For such ultrathin films direct experimental quantification of size effects are complicated by extrinsic effects such as leakage and therefore methods other than the traditional $P-E$ hysteresis loop have been reported to characterize the stability of the polar state. ${ }^{1,7}$ This letter presents experimental measurements of the switched polarization in PZT films of thickness down to $4 \mathrm{~nm}$, in the presence of real electrodes.

A 70-nm-thick SRO layer was grown on STO at $650{ }^{\circ} \mathrm{C}$ followed by the PZT layer via pulsed laser deposition. The deposition was carried out at 100 mTorr of oxygen and the sample was cooled down from growth temperature $1 \mathrm{~atm}$ of oxygen. In order to avoid complications from $90^{\circ}$ domain formation the PZT films were grown in a thickness range from 4 to $80 \mathrm{~nm}$, for which they are entirely $c$-axis oriented. The switchable polarization was measured using a Radiant Technologies Precision Premier system at $16 \mathrm{kHz}$ (hysteresis loops) and an AFM based pulsed probing technique with conductive Pt-Ir tips was employed to measure the pulsed polarization. ${ }^{16}$

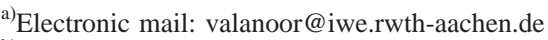

${ }^{b}$ Present address: Department of Materials Science and Engineering, University of California, Berkeley, CA.
}

We focused on the PZT $(0 / 20 / 80)$ composition, since it has an in-plane lattice parameter of $3.94 \AA{ }^{17}$ which is closely lattice matched to $\operatorname{SRO}(3.93 \AA)$. Figure $1(\mathrm{a})$ is a low magnification TEM image of a $4 \mathrm{~nm}$ (nominal) thick film; the interfaces between SRO and PZT are sharp, identified as dashed lines in the high resolution image, Fig. 1(b). The electrode-ferroelectric interface shows a significantly reduced dislocation density, attributed to the small lattice mismatch $(0.7 \%)$ at growth temperature of $600{ }^{\circ} \mathrm{C}$. The inset to Fig. 1(b) is a selected area optical diffraction of the highresolution image, which yields a quantitative tetragonality of 1.05 .

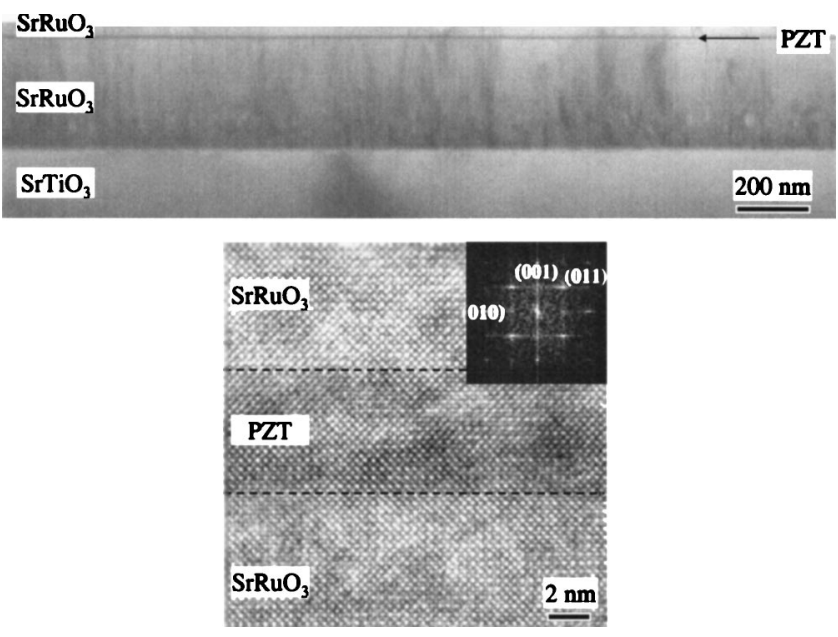

FIG. 1. Structural characterization of ultrathin $\mathrm{PbZr}_{0.2} \mathrm{Ti}_{0.8} \mathrm{O}_{3}$ films with high resolution transmission electron microscopy (HRTEM). (a) Crosssection TEM of $4 \mathrm{~nm}$ PZT film with top and bottom $\mathrm{SrRuO}_{3}$ (SRO) layers grown on $\mathrm{SrTiO}_{3}$ (STO). The image shows that the $4 \mathrm{~nm}$ PZT layer is continuous over several microns length. (b) HRTEM of the same sample. It shows the interfaces are sharp and clean, without misfit dislocations. Inset shows selected area optical diffraction obtained from the HRTEM image. 

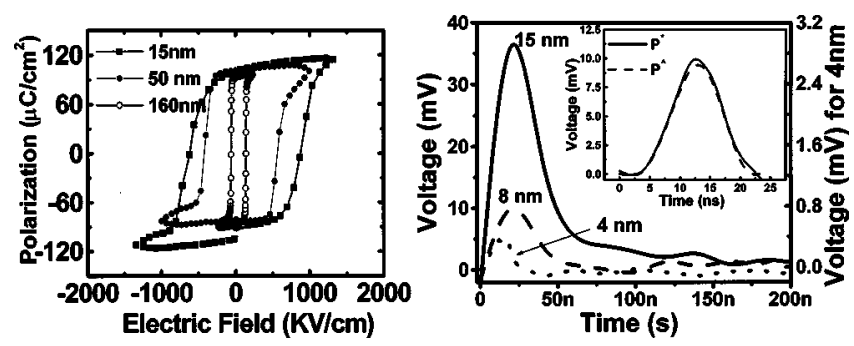

FIG. 2. Ferroelectric measurements as a function of film thickness. (a) Hysteresis loops for 15-, 50-, and 160-nm-thick PZT films. The loops are sharp and well saturated down to $15 \mathrm{~nm}$ with $2 P_{r} \sim 150 \mu \mathrm{C} / \mathrm{cm}^{2}$. (b) Current transients for the 15 (solid), 8 (dashed), and $4 \mathrm{~nm}$ (dotted) film, respectively, measured with the AFM based pulsed probing technique. The applied field was $2750 \mathrm{kV} / \mathrm{cm}$ for the 8 - and 15 -nm-thick film, while $3250 \mathrm{kV} / \mathrm{cm}$ was applied to the $4 \mathrm{~nm}$ film. A sharp drop in the signal strength as seen from the vertical scale is observed. The inset shows switched (solid) $P^{*}$ and nonswitched (dashed) $P^{\wedge}$ response for the 4-nm-thick film.

Figure 2(a) is a set of hysteresis loops measured at $16 \mathrm{kHz}$ for $15-, 50-$, and 160-nm- (for comparison) thick films. The $2 P_{r}$ value obtained from the loop for the 15 -nm-thick film is $\sim 150 \mu \mathrm{C} / \mathrm{cm}^{2}$, which is in agreement with theoretically predicted values for this composition of PZT with an in-plane compressive strain. ${ }^{10,17,18}$ Additionally a systematic increase in the coercive field is observed as the thickness is scaled down to $15 \mathrm{~nm}$, in agreement with previous reports. ${ }^{1,2,19}$ However for sub-10-nm-thick films the hysteresis loops were leaky, and not credible. Therefore we measured the polarization under pulsed probing conditions using an AFM based test setup. ${ }^{16}$ In order to reduce short circuit paths and to allow fast rise times, capacitors with submicron lateral dimensions were fabricated using a modified lift-off technique in which the photoresist was deliberately underdeveloped. By making contact to 0.4 by $0.4 \mu \mathrm{m}^{2}$ pads, we recorded the switched $\left(P^{*}\right)$ and nonswitched polarization $\left(P^{\wedge}\right)$ to obtain the switchable polarization $(\Delta P)$. Figure 2(b) plots the switching transients of the 15 and $8 \mathrm{~nm}$ at an applied field of $2750 \mathrm{kV} / \mathrm{cm}$ and transients measured for the $4 \mathrm{~nm}$ film at $3250 \mathrm{kV} / \mathrm{cm}$, respectively. The switched charge (i.e., area under the transient) for the 15 and $8 \mathrm{~nm}$ films were calculated to be 145 and $70 \pm 5 \mu \mathrm{C} / \mathrm{cm}^{2}$, respectively. A higher field was necessary for the $4 \mathrm{~nm}$ film to obtain a fully switched transient. Integration of the $\Delta P$ transient, yielded a switched charge of $11 \pm 3 \mu \mathrm{C} / \mathrm{cm}^{2}$. This is also evident in the change of the vertical scale in the signal response recorded by the oscilloscope. For the $15 \mathrm{~nm}$ film it is approximately $40 \mathrm{mV}$ (left axis) while for the $4 \mathrm{~nm}$ film it has dropped to $0.5 \mathrm{mV}$ (right axis). The inset to Fig. 2(b) is a plot of the $P^{*}$ and $P^{\wedge}$ transients for the $4 \mathrm{~nm}$ film. The difference between the $P^{*}$ and $P^{\wedge}$ is minute, indicative of a very low magnitude of the switched charge. These results demonstrate that down to $15 \mathrm{~nm}$ there is no observable drop in the switched polarization. Below $15 \mathrm{~nm}$, it progressively decreases to approximately $11 \mu \mathrm{C} / \mathrm{cm}^{2}$ for the $4 \mathrm{~nm}$ film.

We now compare the experimentally measured thickness dependence with that predicted from theory. In Fig. 3 we show the normalized polarization (termed as the order parameter) as a function of film thickness normalized to the correlation length, $\xi(d / \xi)$. The value of $\xi$ for this particular composition was calculated to be $2.4 \mathrm{~nm}$ using parameters from $\mathrm{Li}^{20}$ and Zembilgotov. ${ }^{10}$ We also plot the predicted thickness dependence, from an intrinsic size effect model $^{9,10,20}$ (solid plot), from the macroscopic depoling field

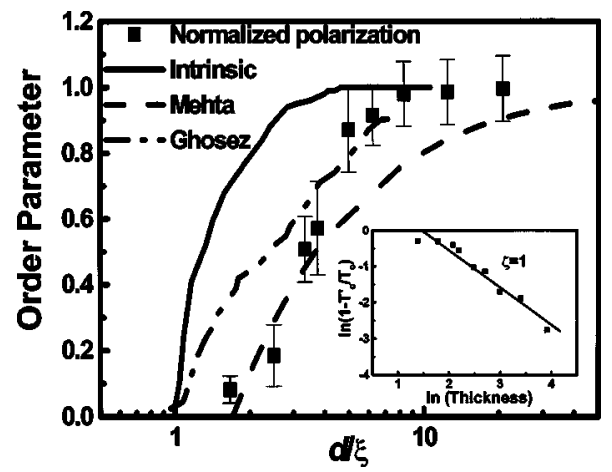

FIG. 3. Comparison of the order parameter as a function of thickness with theoretical predictions. The solid plot shows the predictions from an intrinsic size effect model, the dashed plot from the macroscopic depoling field model and the dotted plot is ab initio depoling field calculations for SRO/ BTO/SRO by Junquera and Ghosez. The squares are experimentally measured switched polarization normalized to the thick film value. The inset plots the calculated $\ln \left[\left(T_{C}^{\text {film }}-T_{C}\right) / T_{C}\right]$ vs $\ln$ (thickness) with a linear fit to the calculation, which reveals a slope $\zeta=1$.

model $^{14,15}$ (dashed plot), and ab initio calculations for $\mathrm{SRO} / \mathrm{BaTiO}_{3} / \mathrm{SRO}$ (dotted plot). The plot shows that the sub-10-nm-thick films lie more closely to the depoling model. However in order to clearly differentiate between the intrinsic size effect and depoling field effect, we need to explore the nature of the critical exponent (or scaling law). Using the approach of Kretschmer and Binder ${ }^{13}$ the Curie temperature of the thin film, $T_{C}^{\text {film }}$, is related to the expected theoretical transition temperature for a film of the same thickness (but without any polarization suppression, $T_{C}$ ), as

$$
\frac{T_{C}^{\mathrm{film}}-T_{C}}{T_{C}} \propto d^{-\zeta},
$$

where $d$ is the thickness of the film and $\zeta$ is the shift exponent, equal to 1 for the case where the suppression is dominated by depolarizing field and $\zeta=2$ for the case where the suppression is dominated by intrinsic effects. ${ }^{13}$ The inset to Fig. 3, which plots

$$
\ln \left(\frac{T_{C}^{\mathrm{film}}-T_{C}}{T_{C}}\right)
$$

as a function of film thickness, is a theoretical prediction of the dependence of $T_{C}$ using an elementary model based on the Landau-Ginzburg-Devonshire (LGD) equation. We have used the formalism of Pertsev et al. ${ }^{10}$ to modify prefactors in the

$$
\begin{aligned}
& \alpha^{\prime}=\alpha-\frac{2 Q_{12} \varepsilon_{M}}{S_{11}+S_{12}}, \\
& \beta^{\prime}=\beta+\frac{4 Q_{12}^{2}}{S_{11}+S_{12}}, \\
& \gamma^{\prime}=\gamma,
\end{aligned}
$$

where $\varepsilon_{M}$ is the misfit strain, $S_{i j}$ is the compliance along the respective crystallographic directions, and $Q_{12}$ is the electrostriction coefficient. To extract $T_{C}^{\text {film }}$, we used the CurieWeiss law and the above modified LGD equations, which correlate the measured polarization to the transition temperature. Our preliminary calculations show a linear fit with a slope of $\sim 1$, suggesting the governing role of depoling fields 
at the electrode-ferroelectric interface in determining the stability of ferroelectricity, in agreement with the theoretical predictions of Ghosez ${ }^{5}$ and Glinchuk. ${ }^{4}$ However to conclusively verify this, accurate experimental determination of $T_{C}$ for a ferroelectric heterostructure with top and bottom electrodes, coupled with a more complex model which takes into account the surface and/or depolarization effects, ${ }^{9,10}$ is needed. Such experiments on the direct experimental determination of $T_{C}$ and the corresponding calculations are ongoing.

This work was supported by NSF MRSEC Contract No. 00-80008 and a NSF US-Europe collaborative Grant No. DMR 02-44288. The work at University of Wisconsin is supported by the NSF under Contract No. DMR-9973801. V.N. acknowledges the Alexander von Humboldt foundation for his stay in FZ-Juelich, Germany. We wish to thank S. Streiffer and $\mathrm{Ph}$. Ghosez for fruitful discussions and critical reading of the manuscript.

${ }^{1}$ A. V. Bune, V. M. Fridkin, S. Ducharme, L. M. Blinov, S. P. Palto, A. V. Sorokin, S. G. Yudin, and A. Zlatkin, Nature (London) 391, 874 (1998). ${ }^{2}$ M. Dawber, P. Chandra, P. B. Littlewood, and J. F. Scott, J. Phys.: Condens. Matter 15, L393 (2003).

${ }^{3}$ P. Ghosez and K. M. Rabe, Appl. Phys. Lett. 76, 2767 (2000).

${ }^{4}$ M. D. Glinchuk, E. A. Eliseev, and V. A. Stephanovich, Physica B 322, 356 (2002).

${ }^{5}$ J. Junquera and P. Ghosez, Nature (London) 422, 506 (2003).
${ }^{6}$ T. M. Shaw, S.-T. McKinstry, and P. C. McIntyre, Annu. Rev. Mater. Sci. 30, 263 (2000).

${ }^{7}$ S. K. Streiffer, J. A. Eastman, D. D. Fong, C. Thompson, A. Munkholm, M. V. R. Murty, O. Auciello, G. R. Bai, and G. B. Stephensonl, Phys. Rev. Lett. 89, 067601 (2002).

${ }^{8}$ T. Tybell, C. H. Ahn, and J.-M. Triscone, Appl. Phys. Lett. 75, 856 (1999).

${ }^{9}$ W. L. Zhong, B. D. Qu, P. L. Zhang, and Y. G. Wang, Phys. Rev. B 50, 12375 (1994).

${ }^{10}$ A. G. Zembilgotov, N. A. Pertsev, H. Kohlstedt, and R. Waser, J. Appl. Phys. 91, 2247 (2002).

${ }^{11}$ C. L. Wang, Y. Xin, X. S. Wang, and W. L. Zhong, Phys. Rev. B 62, 11423 (2000).

${ }^{12}$ N. Yanase, K. Abe, N. Fukushima, and T. Kawakubo, Jpn. J. Appl. Phys., Part 1 38, 5305 (1999).

${ }^{13}$ R. Kretschmer and K. Binder, Phys. Rev. B 20, 1065 (1979).

${ }^{14}$ I. P. Batra, P. Wurfel, and B. D. Silverman, J. Vac. Sci. Technol. 10, 687 (1973).

${ }^{15}$ R. R. Mehta, B. D. Silverman, and J. T. Jacobs, J. Appl. Phys. 44, 3379 (1973).

${ }^{16}$ S. Prasertchoung, V. Nagarajan, Z. Ma, R. Ramesh, J. S. Cross, and M. Tsukada, Appl. Phys. Lett. 84, 3130 (2003).

${ }^{17}$ Landolt-Börnstein, Numerical Data and Functional Relationships in Science and Technology, Vol. 16, edited by K.-H. Hellwege and A. M. Hellwege (Springer, Berlin, 1981).

${ }^{18}$ M. J. Haun, E. Furman, S. J. Jang, and L. E. Cross, Ferroelectrics 99, 45 (1989).

${ }^{19}$ N. A. Pertsev, J. R. Contreras, V. G. Kukhar, B. Hermanns, H. Kohlstedt, and R. Waser, Appl. Phys. Lett. 83, 3356 (2003).

${ }^{20}$ S. Li, J. A. Eastman, J. M. Vetrone, C. M. Foster, R. E. Newnham, and L. E. Cross, Jpn. J. Appl. Phys., Part 1 36, 5169 (1997). 